\title{
ANALYSis AND OPTIMIZATION OF A DOUBle-SIDED, AIR-CORED TUBUlar GENERATOR
}

\author{
Liang Guo ${ }^{1}$, He Zhang ${ }^{2}$, Jing Li $^{2}$, Michael Galea ${ }^{2}$, and Chris Gerada ${ }^{2}$ \\ ${ }^{1}$ Faculty of Mechanical Engineering \& Automation, Zhejiang Sci-Tech University, Hangzhou, Zhejiang \\ ${ }^{2}$ PEMC group, Faculty of Engineering, University of Nottingham, Nottingham, United Kingdom
}

In this paper, the design and development of a double-sided, air-cored tubular generator (DSTG) is presented. The characteristics of air-cored, slot-less structures are investigated in terms of power density and efficiency. This is highly desirable for small and medium-sized renewable energy conversion systems. In this work, an analytical tool based on the magnetic vector potential method is built in order to achieve a fast but accurate method by which to analyze the machine. The analytical model is validated by finite element analysis. Further design improvements, based on the analytical and FE models are then suggested in order to enhance the performance of the DSTG. The final design of the DSTG is validated by test results from a developed prototype of the DSTG.

Index Terms - Double-sided, tubular generator, analytical modeling, optimization

\section{INTRODUCTION}

I $\mathrm{t}$ is generally known, that slotted, tubular machines can achieve very good electro-magnetic performances [1 - 4] with extremely high torque and power density values, with however mixed results in terms of force ripple and cogging forces. Air-cored, tubular generators on the other hand, can achieve excellent force ripple values and a lower moving mass, which effectively enhances the dynamic stability and the generated power quality. Air-cored tubular generators can be found in a number of applications, such as energy conversion systems of ambient vibrations, free-piston engines and tidal wave energy conversion systems [5, 6].

The relatively large equivalent air-gap of slot-less configurations limits the generator's power density. In order to overcome this challenge, several methods have been proposed in literature. In [7], a moving-coil generator is proposed where an equivalent magnetic circuit model of the generator with one phase was established and the basic characteristics were analyzed. In [8, 9], a three phase topology in motoring operation is proposed. The magnetic fields generated by the PMs and the armature are analyzed and the variations of forces with the main dimensions were obtained.

In this paper, a multi air-gap, tubular generator with double, permanent magnet (PM) arrays, as shown in Fig.1 is proposed. Analytical expressions of the flux density distributions in the proposed DSTG are established by using the magnetic vector potential method [10, 11]. Calculation methods for the model parameters are also provided, which can be used as an effective tool for optimization and for the control system. The analytical model is used to accurately derive and represent the induced voltages, the generated force and the overall efficiency, which are the main characteristics of the generator considered in this work. The accuracy of the analytical results is verified by finite element (FE) analysis. An experimental prototype of the DTSG is then manufactured and the experimental data resulting from testing, used to validate both the analytical and the FE methods.

Manuscript received March 20, 2015 (date on which paper was submitted for review). Corresponding author: He Zhang (e-mail: he.zhang @ nottingham. ac.uk).

Digital Object Identifier inserted by IEEE

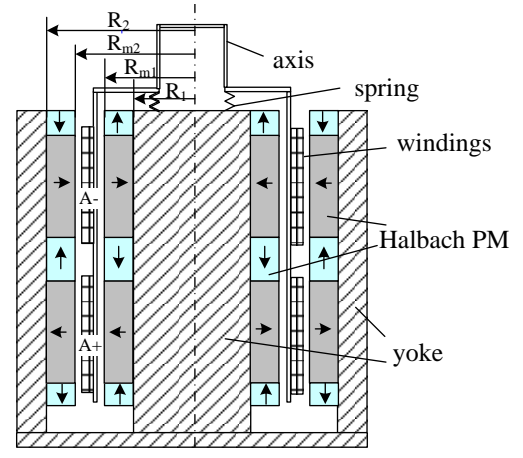

Fig. 1 Construction of the proposed DSTG

\section{ANALyticAl Modeling OF The DSTG}

\section{A. Flux Density Distribution Due to PMs}

Analytical expressions of the magnetic field distributions for a single-sided, tubular machine with quasi-halbach PMs are introduced in $[12,13]$. To simplify the model for the proposed DSTG, the flux density in the DSTG can be assumed as the mathematical summation of two single-sided tubular machines, while assuming that there is minimum and negligible magnetic saturation. Thus, the outer single-sided tubular machine can be considered as a tubular machine with external quasi-halbach PMs as shown in Fig. 2, where region $I$ represents the air-gap/winding region and region II caters for the PM region. The other part of the machine is considered as a tubular machine with internal quasi-halbach PMs, as shown in Fig. 3. The following assumptions are considered:

1) The relative permeability of PMs $\mu_{r}=1$;

2) The effects of eddy currents are neglected;

3) The z-axis length of the PM array is infinite so that the end-effect can be neglected.

For the two different regions, the vector magnetic potential can be described as shown in (1)

$$
\left.\begin{array}{lr}
\nabla^{2} \mathbf{A}=0 & \text { in region I } \\
\nabla^{2} \mathbf{A}=-\mu_{0} \nabla \times \mathbf{M} & \text { in region II }
\end{array}\right\}
$$

For the configuration of Fig. 2, the magnetization vector $\mathbf{M}_{e}$ and the field vectors $\mathbf{B}_{e}$ and $\mathbf{H}_{e}$ in region $I$ and region $I I$ must satisfy (2). 


$$
\begin{aligned}
& \mathbf{M}_{e}=M_{r e} e_{r}+M_{z e} e_{z} ;\left.B_{\mathrm{I} r e}\right|_{R_{m 2}}=\left.B_{\mathrm{II} r e}\right|_{R_{m 2}} \\
& \left.H_{\mathrm{I} z e}\right|_{R_{m 2}}=\left.H_{\mathrm{II} z e}\right|_{R_{m 2}} ;\left.B_{\mathrm{I} z e}\right|_{R_{1}}=0 ;\left.H_{\mathrm{II} z e}\right|_{R_{2}}=0
\end{aligned}
$$

where $M_{r e}=\sum_{n=1,2, \cdots}^{\infty} M_{r n} \sin \left(m_{n} z\right), M_{z e}=\sum_{n=1,2, \cdots}^{\infty} M_{z n} \cos \left(m_{n} z\right)$, $M_{r n}=\frac{4 B_{r}}{\mu_{0} \tau m_{n}} \sin \frac{m_{n} \tau}{2} \sin \frac{m_{n} \tau_{m r}}{2}, \quad M_{z n}=\frac{4 B_{r}}{\mu_{0} \tau m_{n}} \sin \frac{m_{n} \tau_{m z}}{2}$, $m_{n}=\frac{(2 n-1) \pi}{\tau}$, and $B_{r}$ is the remanence of the magnets, $\tau$ is the pole pitch, $\tau_{m r}$ is the width of the radially-magnetized PMs and $\tau_{m z}$ is the width of axially-magnetized PMs.

For the model shown in Fig. 3, the magnetization $\mathbf{M}_{i}$, the field vectors $\mathbf{B}_{i}$ and $\mathbf{H}_{i}$ in region $I$ and region $I I$ satisfy the following equations.

$$
\begin{aligned}
& \mathbf{M}_{i}=M_{r i} e_{r}+M_{z i} e_{z} ;\left.B_{\mathrm{I} r i}\right|_{R_{m 1}}=\left.B_{\mathrm{II} r i}\right|_{R_{m 1}} ; \\
& \left.H_{\mathrm{I} z i}\right|_{R_{m 1}}=\left.H_{\mathrm{II} z i}\right|_{R_{m 1}} ;\left.H_{\mathrm{II} z i}\right|_{R_{1}}=0 ;\left.B_{\mathrm{I} z i}\right|_{R_{2}}=0 ;
\end{aligned}
$$

where $M_{z i}=-M_{z e}, M_{r i}=M_{r e}$.

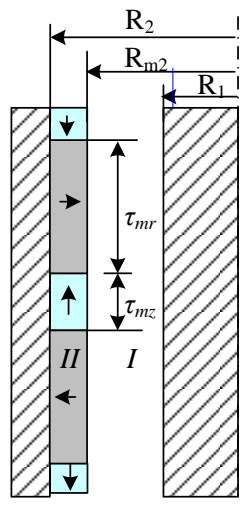

Fig.2 one with external PMs

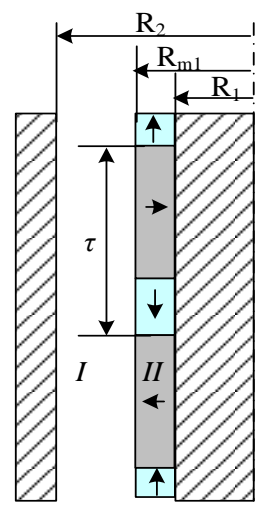

Fig. 3 one with internal PMs
Combining (1) with conditions (2) and (3), the flux density distribution components in region $I$ can be derived as:

$$
\begin{aligned}
B_{I r}(r, z) & =B_{I r e}(r, z)+B_{I r i}(r, z) \\
& =\sum_{n=1,2, \cdots}^{\infty}\left[a_{1 n} I_{1}\left(m_{n} r\right)+b_{1 n} K_{1}\left(m_{n} r\right)\right] \sin \left(m_{n} z\right) \\
B_{I z}(r, z) & =B_{I z e}(r, z)+B_{I z i}(r, z) \\
& =\sum_{n=1,2, \cdots}^{\infty}\left[a_{1 n} I_{0}\left(m_{n} r\right)-b_{1 n} K_{0}\left(m_{n} r\right)\right] \cos \left(m_{n} z\right)
\end{aligned}
$$

where $a_{1 n}=a_{1 n e}+a_{1 n i}, b_{1 n}=b_{1 n e}+b_{1 n i}$. As shown in [12], the parameters for flux density generated by the external PMs configuration are defined by $a_{\text {lne }}$ and $b_{\text {Ine }}$, which can be derived according to the conditions described by (2). The parameters for flux density generated by the internal PMs configuration are defined by $a_{I n i}$ and $b_{1 n i}$, which can be derived according to the conditions described by (3). $I_{0}(\mathrm{x}, \mathrm{y})$ and $K_{0}(\mathrm{x}, \mathrm{y})$ are modified Bessel functions of the first and second kind of the 0th order, respectively. $I_{l}(\mathrm{x}, \mathrm{y})$ and $K_{l}(\mathrm{x}, \mathrm{y})$ are modified Bessel functions of the first and second kind of the 1st order, respectively.

\section{B. Flux Density Distribution Due to Armature Current}

The flux distribution in region $I$ and region $I I$, due to the armature current is shown in Fig.4a, where $R_{\text {middle }}=\left(R_{c 1}+R_{c 2}\right) / 2$. In order to simplify the analysis, equivalent configurations such as shown in Fig. 4b and Fig. 4c can be safely assumed. This is true, when considering the assumption that the field lines are always perpendicular to the centerline of the windings. For the configurations of Fig. $4 \mathrm{~b}$ and Fig. $4 \mathrm{c}$, the current distribution of phase $a$ can be expressed by (6), while the conditions of field vectors $\mathbf{B}_{a}$ and $\mathbf{H}_{\boldsymbol{a}}$ in region $I$ and region II can be expressed as described by (7) and (8), respectively.

$$
\begin{gathered}
J_{a}=\sum_{n=1,2, \cdots}^{\infty} J_{a n} \sin \left(m_{n} z_{a}\right) \\
\left.B_{\mathrm{I} z a}\right|_{R_{c 1}}=\left.B_{\mathrm{II} z a}\right|_{R_{c 1}} ;\left.B_{\mathrm{II} z a}\right|_{R_{m i d a l e}}=0 ;\left.B_{\mathrm{I} z a}\right|_{R_{1}}=0 \\
\left.B_{\mathrm{I} z a}\right|_{R_{c 2}}=\left.B_{\mathrm{II} z a}\right|_{R_{c 2}} ;\left.B_{\mathrm{II} z a}\right|_{R_{\text {middle }}}=0 ;\left.B_{\mathrm{I} z a}\right|_{R_{2}}=0
\end{gathered}
$$

where $J_{a n}=\frac{4 J_{0}}{\tau m_{n}} \sin \frac{m_{n} w_{c}}{2}, z_{a}$ is Z-axis position related to the phase $a$ windings, $w_{c}$ is the z-axis height of the winding and $J_{0}$ is the current density.

For these conditions, for a single phase $a$, the flux density components $B_{\mathrm{I} r a}$ and $B_{\mathrm{I} z a}$ in the region $I$ can be expressed as in (9) and (10). The flux density components $B_{\mathrm{II} r a}$ and $B_{\mathrm{II} z a}$ in region $I I$ can be expressed as (11) and (12).

$$
\begin{gathered}
B_{\text {Ira }}\left(r, z_{a}\right)=-\sum_{n=1,2, \cdots}^{\infty}\left[a_{1 a n} I_{1}\left(m_{n} r\right)+b_{1 a n} K_{1}\left(m_{n} r\right)\right] \cos \left(m_{n} z_{a}\right) \\
B_{I z a}\left(r, z_{a}\right)=\sum_{n=1,2, \cdots}^{\infty}\left[a_{1 a n} I_{0}\left(m_{n} r\right)-b_{1 a n} K_{0}\left(m_{n} r\right)\right] \sin \left(m_{n} z_{a}\right) \\
B_{\text {IIra }}\left(r, z_{a}\right)=-\sum_{n=1,2, \cdots}^{\infty}\left\{\begin{array}{l}
{\left[F_{\text {Aan }}\left(m_{n} r\right)+a_{11 a n}\right] I_{1}\left(m_{n} r\right)} \\
\left.\left.+-F_{B a n}\left(m_{n} r\right)+b_{11 a n}\right] K_{1}\left(m_{n} r\right)\right\}
\end{array}\right\} \cos \left(m_{n} z_{a}\right) \\
B_{\text {IIza }}\left(r, z_{a}\right)=\sum_{n=1,2, \cdots}^{\infty}\left\{\begin{array}{l}
{\left[F_{\text {Aan }}\left(m_{n} r\right)+a_{11 a n}\right] I_{0}\left(m_{n} r\right)} \\
\left.+\left[F_{\text {Ban }}\left(m_{n} r\right)-b_{11 a n}\right] K_{0}\left(m_{n} r\right)\right\} \sin \left(m_{n} z_{a}\right)
\end{array}\right.
\end{gathered}
$$

where $a_{\text {Ian }}(\mathrm{)}) b_{\text {Ian }}(\mathrm{)}), a_{\text {IIan }}(\mathrm{)}), b_{\text {IIan }}\left(\mathrm{)}, F_{\text {Aan }}(\mathrm{)})\right.$ and $F_{\text {Ban }}(\mathrm{)}$ can be derived as shown in [13].

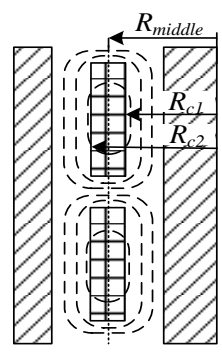

(a)

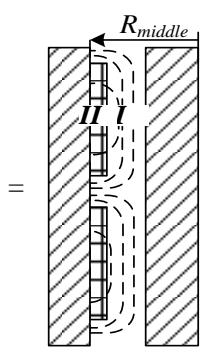

(b)

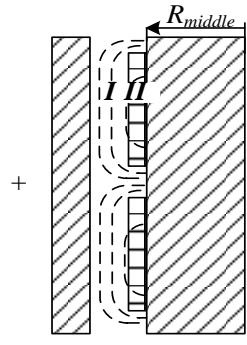

(c)
Fig.4 Armature field flux distribution and equivalent solutions

\section{Induced voltage and force calculation}

Two quality indicators for any generator are the induced voltages and the generated force. The resulting efficiency is dependent on these two aspects, while any harmonic components affect the power quality and dynamic stability of the system. Considering the influence of the armature field, the coil flux-linkage in any one winding can be expressed as 


$$
\phi_{i}=N_{1} \int_{z_{i}-\frac{w_{c}}{2}}^{z_{i}+\frac{w_{c}}{2}} \int_{R_{c 2}}^{R_{c 1}} 2 \pi r\left(\sum_{n=1}^{\infty} B_{I r}+\sum_{m=1}^{\text {phase }} \sum_{n=1}^{\infty} B_{I I r m}\right) d r d z
$$

where $N_{l}$ is the number of the turns of the windings, $z_{i}$ is the $\mathrm{z}$ axis position of the windings centre-height, $B_{I r}$ is the PMs-due flux density, $B_{\text {IIrm }}$ is the flux density generated by the $m$-phase windings and phase is the number of phases. Since the position of the armature field $B_{\text {IIrm }}$ is fixed relative to the windings and the windings are full-pitch, the voltage across one phase can be calculated as shown below.

$$
\begin{aligned}
e & =-d \phi / d t \\
& \left.=-\sum_{n=1}^{\infty} \frac{4 \pi p K_{d n} N_{1} v}{\left(R_{c 1}-R_{c 2}\right)} \mid \begin{array}{l}
\int_{R_{c 2}}^{R_{c 1}} r K_{I r n} d r \sin m_{n}\left(z-\frac{\tau}{2}\right) \\
+\int_{R_{c 2}}^{R_{c 1}} r \sum_{m=1}^{\text {phase }} K_{\text {IIrmn }} d r
\end{array}\right)
\end{aligned}
$$

where $K_{d n}=\sin \left(m_{n} w_{c} / 2\right) /\left(m_{n} w_{c} / 2\right)$

$K_{I r n}=a_{1 n} I_{1}\left(m_{n} r\right)+b_{1 n} K_{1}\left(m_{n} r\right), p$ is the pole-pairs, $v$ is the linear velocity, $m$ present the parameters of $m$-phase and

$K_{I I r m n}=\left[F_{A m n}\left(m_{n} r\right)+a_{11 \mathrm{~m} n}\right] I_{1}\left(m_{n} r\right)+\left[-F_{B m n}\left(m_{n} r\right)+b_{1 \operatorname{Im} n}\right] K_{1}\left(m_{n} r\right)$.

Considering that $F=\int_{v}(J \times B) d v$, where $J$ is the current density, the Lorentz force generated in one winding is

$$
F=-\int_{z_{i}-\frac{w_{c}}{2}}^{z_{i}+\frac{w_{c}}{2}} \int_{R_{c 2}}^{R_{c 1}} 2 \pi r J\left(\sum_{n=1}^{\infty} B_{I r}+\sum_{m=1}^{\text {phase }} \sum_{n=1}^{\infty} B_{I I r m}\right) d r d z
$$

Substituting $(4,5)$ and $(11,12)$ into $(15)$, then the thrust force of one phase can be calculated as the following:

$$
\left.F=-\sum_{n=1}^{\infty} \frac{4 \pi p K_{d n} N_{1} i}{\left(R_{c 1}-R_{c 2}\right)} \mid \begin{array}{l}
\int_{R_{c 2}}^{R_{c 1}} r K_{I r n} d r \sin m_{n}\left(z-\frac{\tau}{2}\right) \\
+\int_{R_{c 2}}^{R_{c 1}} r \sum_{m=1}^{p h a s e} K_{I I r m n} d r
\end{array}\right\}
$$

where $i$ is the current. The total thrust force of a multi-phase generator can be derived by adding the forces generated by each phase windings.

\section{Validation by FEM}

In order to verify the analytical models presented above, a FE model of the single phase DSTG is built. The main dimensions of the generator are given in Table I. The model is used to simulate rated operation conditions, with $80^{\circ} \mathrm{C}$ in the windings/PMs regions and $25^{\circ} \mathrm{C}$ ambient temperature, derived by a lumped parameter, thermal network model [14] .The comparison between the results calculated by the FE method and the analytical method are shown in Fig. 5 - Fig. 7.

Fig. 5 illustrates the radial component $B_{r}$ of the flux density distribution in the air-gap at radius $R=\left(R_{m 2}+R_{c 2}\right) / 2$. Since the number of pole pairs of the armature is two, the flux density in the range of $[50 \mathrm{~mm}, 250 \mathrm{~mm}]$ is the combination result of the PM armature fields. The flux density in the range of $[250 \mathrm{~mm}$, $550 \mathrm{~mm}$ ] is due only to the PMs. The results show that the analytical results are in very good agreement with the FE results, within the full calculation region, except for the end parts. The difference between the two results comparison is approximately a $0.01 \mathrm{~T}$ discrepancy.

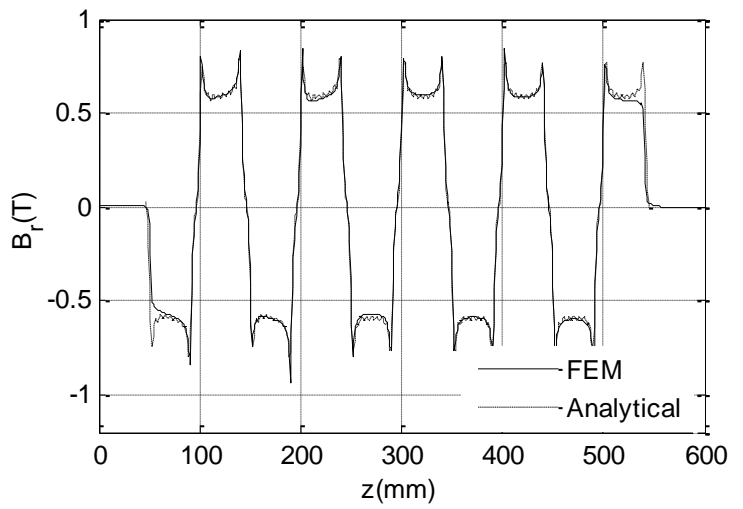

Fig.5 Flux density distribution in air-gap

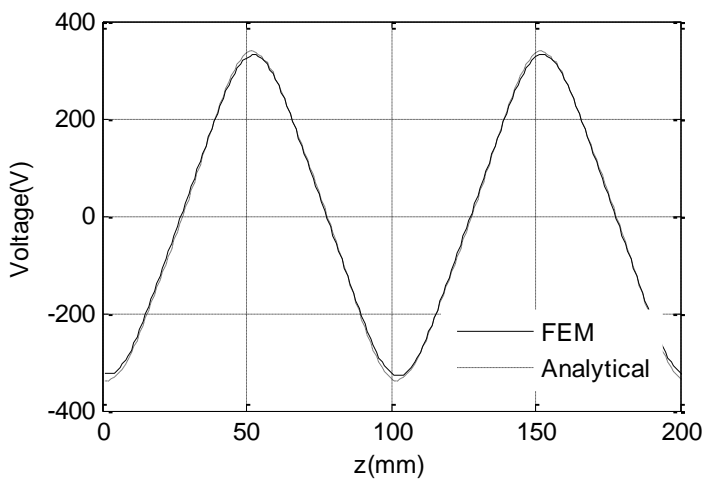

Fig.6 Induced voltage with $J=4 \mathrm{~A} / \mathrm{mm}^{2}$

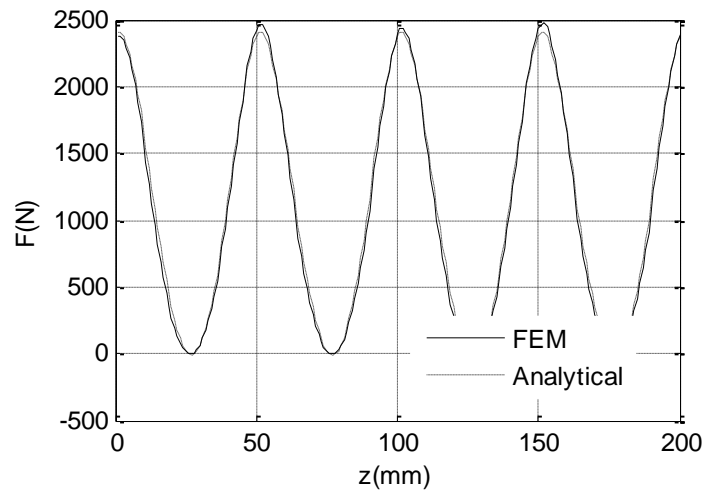

Fig.7 Thrust force with $J=4 \mathrm{~A} / \mathrm{mm}^{2}$

The phase voltage curves shown in Fig. 6 and the thrust force shown in Fig. 7 also prove the accuracy of the analytical results. The voltage difference between the FE results and the analytical method results is less than $2.7 \%$ and the thrust differens by only about $1 \%$, which validates the analytical modelling of the DSTG

TABLE I

MAIN DIMENSIONS OF THE DSTG ( UNIT: mm)

\begin{tabular}{ccccccc}
\hline \hline$R_{2}$ & $R_{m 2}$ & $R_{c 2}$ & $R_{c 1}$ & $R_{m 1}$ & $R_{1}$ & $\tau$ \\
\hline 110 & 102 & 101 & 91 & 89 & 81 & 50 \\
\hline \hline
\end{tabular}

\section{OPTIMIZATION OF THE DSTG}

In this section, the machine's sensitivity to its geometrical dimensions, relative to voltage and efficiency is investigated. An optimized machine design is then proposed with the main objective being to reduce the voltage harmonics and improve the efficiency. 


\section{A. The Influence of Dimensions on the Induced Voltage}

The variations of the voltage with the machine dimensions are analyzed while assuming $R_{2}$ to be constant. The result proves that increasing the PMs thickness $h_{p m}$ (where $h_{p m}=R_{2^{-}}$ $\left.R_{m 2}=R_{m 1}-R_{I}\right)$ and the windings thickness $h_{c}\left(h_{c}=R_{c 2}-R_{c l}\right)$ can enhance the amplitude of the voltage at the cost of reduced active material utilization. Unfortunately, harmonic content is also increased.

The variations of the voltage and harmonic components relative to the machine geometry are illustrated in Fig. 8 and Fig. 9 respectively. A voltage maximum value is obtained when $\tau_{m r} / \tau$ is 0.8 , as shown in Fig. 8, where $\tau_{m r}$ is the width of the PM with radial magnetization. The optimal result in terms of harmonic presence is achieved when $\tau_{m r} / \tau$ is $0.5 \sim 0.6$, as shown in Fig. 9.

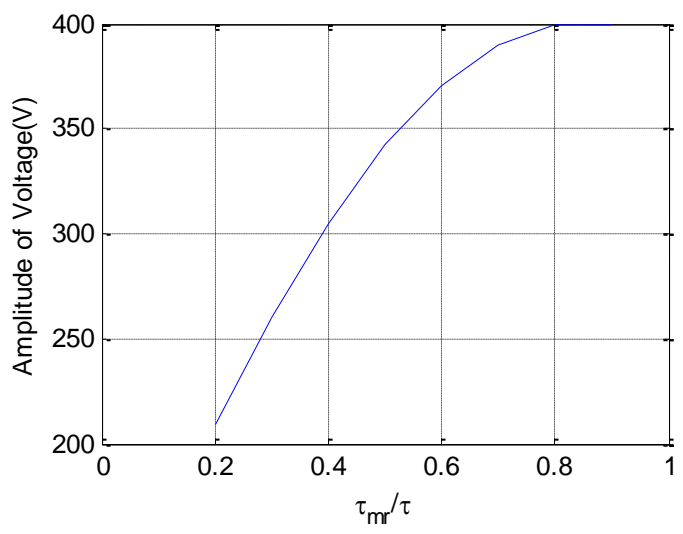

Fig. 8 The variation of EMF with $\tau_{m r} / \tau$

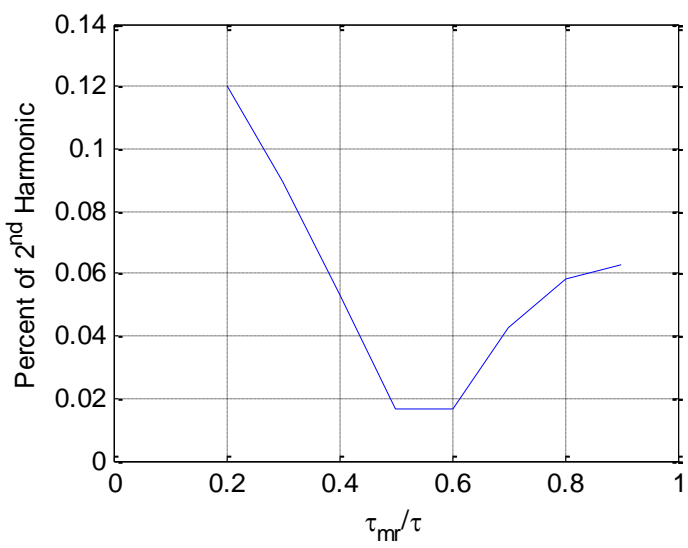

Fig. 9 The variation of harmonics with $\tau_{m r} / \tau$

\section{B. The Influence of Dimensions on Efficiency}

The sensitivity of the efficiency of the DSTG to the machine dimensions is illustrated in Fig. 10 and Fig. 11. From the analytical results, it can be found that the efficiency increases for increments of $h_{m}$, but decreases when $h_{c}$ is increased. The optimal efficiency value is found to be when $\tau_{m r} / \tau$ is 0.8 .

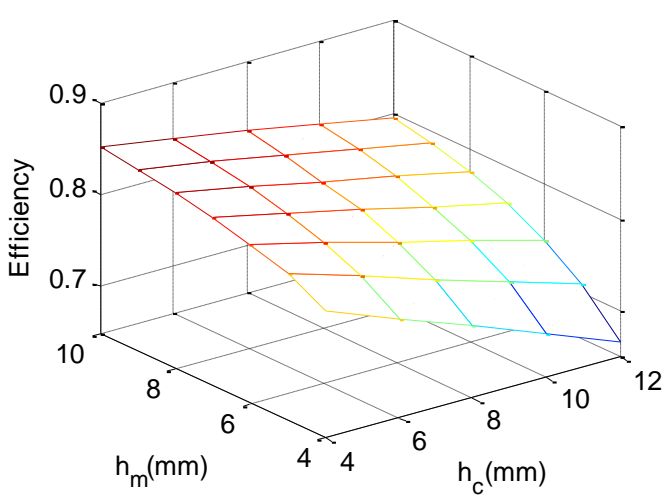

Fig. 10 The variation of efficiency with $h_{p m}$ and $h_{c}$

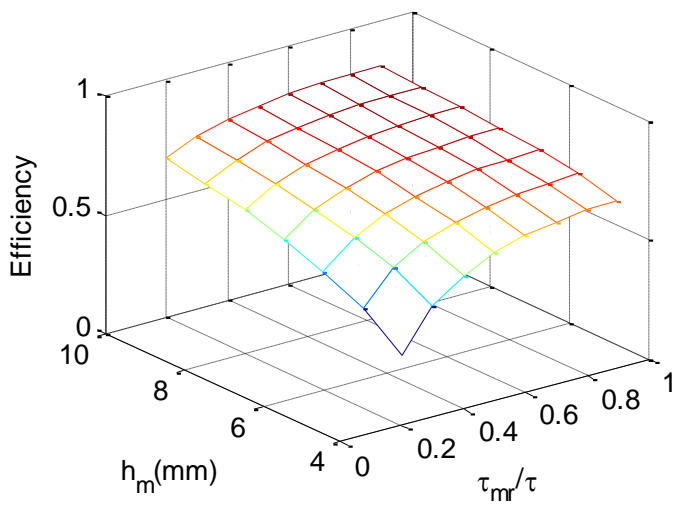

Fig. 11 The variation of efficiency with $\tau_{m r} / \tau_{c}$

\section{Optimised machine}

Table II illustrates how the DTSG performance varies for the different machine dimensions considered in the above optimization exercise. It can be observed that for a constant copper loss, the best overall performances is achieved with $\tau_{m r} / \tau=0.6$. At this point, efficiency is $2 \%$ higher than that of the DSTG with $\tau_{m r} / \tau=0.5$, while also achieving an improvement in the harmonic content of the phase voltage. However, if the harmonic content is not considered, then the maximum efficiency is achieved when $\tau_{m r} / \tau$ is set to 0.8 .

TABLE II

CHARACTERISTICS OF THE DSTG WiTH OPTIMIZED DiMENSIONS

\begin{tabular}{crccc}
\hline \hline$\tau_{m r}(m m)$ & $\tau_{m r} / \tau$ & Efficiency $(\%)$ & Voltage $(V)$ & $2^{\text {nd }}$ harmonic $(\%)$ \\
\hline 25 & 0.5 & 75.66 & 342 & 1.67 \\
30 & 0.6 & 77.54 & 370 & 1.65 \\
40 & 0.8 & 79.18 & 400 & 5.86 \\
\hline \hline
\end{tabular}

\section{PROTOTYPE AND EXPERIMENTS}

To validate the analytical models, a prototype of the DSTG is manufactured based on the parameters in Table III, for the maximum possible efficiency, i.e. when $\tau_{m r} / \tau=0.8$. In order to test the dynamic performance of the prototype, a linear motor is used to drive the DSTG's mover reciprocally. An S-type force sensor and an optical fibre-bragg sensor system are used to detect the force and position signals. The prototype and the experimental rig are illustrated in Fig. 12. 
TABLE III

MAIN DiMENSIONS OF THE PROTOTYPE (UNIT: mm)

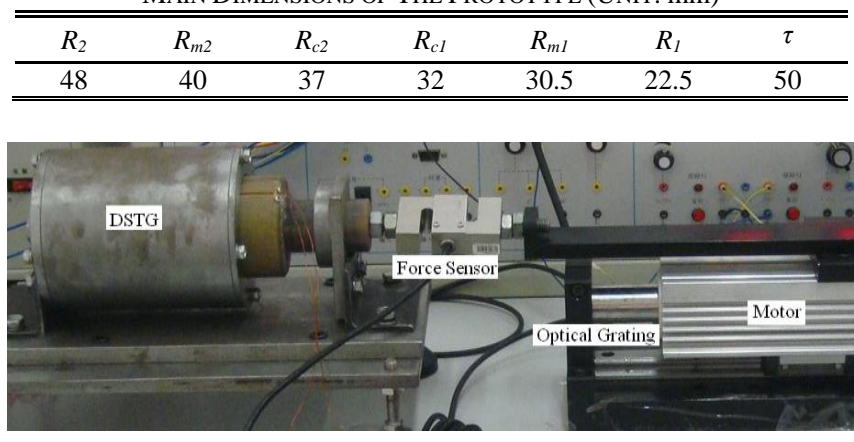

Fig.12 The prototype experiment system

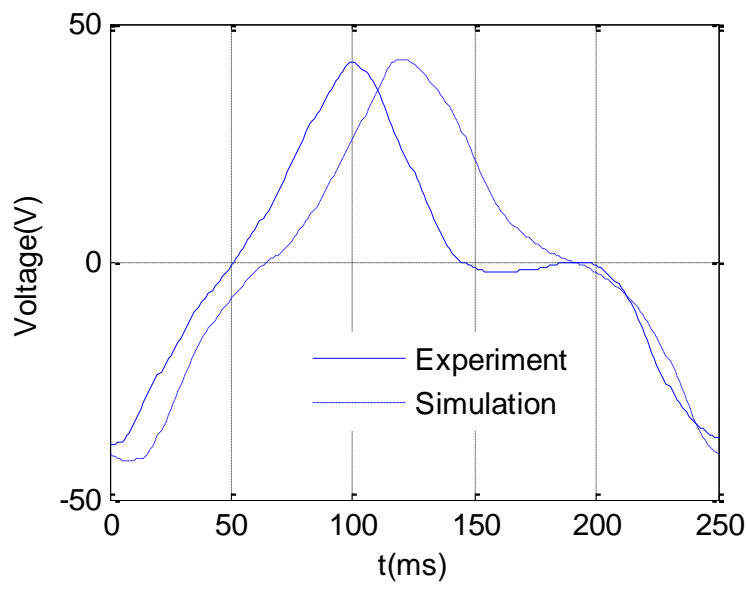

Fig.13 The output voltage

The same operating conditions for the above analysis are considered with the experimental set-up. For a translational stroke of $\pm 20 \mathrm{~mm}$ and with a $303 \Omega$ load resistance, the output voltage of the prototype is measured and this is shown in Fig. 13. The simulation results are in good agreement with the test results with some discrepancy, which is mainly due to measurement errors and any manufacturing factors. A difference in the results between the FE and the experimental tests of less than $2 \%$ has been achieved, which therefore validates the proposed analytical method and the worthiness of the proposed DTSG.

\section{CONCLUSION}

In this paper, analytical models that consider the magnetic field distribution in a slot-less, DSTG have been developed and validated with FE and experimental results. Considering the voltage and thrust force equations, the relationships between the main machine dimensions and the machine performance were investigated. The optimal design for an improved machine performance is obtained based on this sensitivity analysis.

The results show that the increment of the thickness of PMs can improve the voltage and the efficiency of the DSTG, while increasing the thickness of the windings can also enhance the voltage but at the cost of degrading the overall efficiency of the DSTG.
The efficiency of the machine can achieve the maximum value when the width of the PMs with radial magnetization is about $80 \%$ of the pole span. Further work will include the development of advanced algorithms to further optimize the design.

\section{ACKNOWLEDGMENT}

This work was supported in part by Zhejiang Provincial Natural Science Foundation of China (Grant No. LY14E070008), the Program for Changjiang Scholars and Innovative Research Team in University (PCSIRT) (Grant No.IRT13097), and "521" Talent Project of Zhejiang Sci-Tech University.

\section{REFERENCES}

[1] M. Galea, G. Buticchi, L. Empringham, L. De Lillo, and C. Gerada, "Design of a high-force-density tubular motor," IEEE Trans. Indus. Appl., vol. 50, pp. 2523-2532, 2014.

[2] B.L.J.Gysen, K.J. Meessen, F.J.H.Paulides, and E.A.Lomonova, "3D analytical and numerical modeling of tubular actuatiors with skewed permanent magnets," IEEE Trans.magn., vol. 47, No.9. pp. 2200-2212, 2011.

[3] L.Huang, H.Yu, M.Hu, C.Liu and B. Yuan, "Research on a tubular primary permanent-magnet linear generator used for wave energy conversins," IEEE Trans.magn., vol. 49, No.5. pp. 1917-1920, 2013.

[4] C.Liu, H. Yu, M.Hu, Q. Liu and S. Zhou, "Detent force reduction in permanent magnet tubular linear generator for direct-driver wave energy conversion," IEEE Trans.magn., vol. 49, No.5. pp. 1913-1916, 2013.

[5] K. Rhinefrank, E.B. Agamoloh, et al. "Novel ocean energy permanent magnet linear generator buoy," Renewable Energy, vol. 31, pp. 12791298, 2006. (空心)

[6] M. G. Park, J. Y. Choi, H.J. Shin, K. Lee and K. Honh. "Electromagnetic analysis and experimental testing of a tubular linear synchronous machine with a double-Sided axially magnetized permanent magnet mover and coreless stator windings by using semianalytical techniques," IEEE Trans.magn., vol. 50, No.11. 2014,8205204.(空心)

[7] Zhaoping Xu, Siqin Chang, "Improved moving coil electric machine for internal combustion liner generator", IEEE Trans. Energy Conv., vol.25, No.2, pp: 281-286, 2010.

[8] Liang Yan, Lei Zhang, Tianyi Wang, Zongxia Jiao,Chin-Yin Chen, IMing Chen. "Magnetic field of tubular linear machines with dual halbach array", Progress In Electromagnetics Research, vol.136, pp: 283-299, 2013

[9] Liang Yan, Lei Zhang, Zongxia Jiao, Hongjie Hu, Chin-Yin Chen, IMing Chen. Armature reaction field and inductance of coreless movingcoil tubular linear machine. IEEE Trans. Indus. Elec., vol. 61 No. 21, pp. 6956-6965,2014

[10] M. Galea, L. Papini, H. Zhang, C. Gerada, and T. Hamiti, "Demagnetisation Analysis for Halbach Array Configurations in Electrical Machines," IEEE Trans.magn, to be published and available on early access, 2015.

[11] L. Guo, H. Zhang, M. Galea, J. Li, W. Lu, and C. Gerada, "Analysis and design of a magnetically levitated planar motor with novel multilayer windings," IEEE Trans.magn, to be published and available on early access, 2015.

[12] Jiabin Wang, David Howe, " Tubular modular permanent-magnet machines equipped with quasi-halbach magnetized magnets-part I: magnetic field distribution, EMF, and thrust force", IEEE Trans. Magn.,vol. 41,No. 9,pp: 2470-2478,2005.

[13] Jiabin Wang, Geraint W. Jewell and David Howe, "A general framework for the analysis and design of tubular linear permanent magnet machines", IEEE Trans. Magn.,vol.35, No.3, pp:1986-2000, 1999.

[14] M. Galea, C. Gerada, T. Raminosoa, and P. Wheeler, "A Thermal Improvement Technique for the Phase Windings of Electrical Machines," IEEE Trans. Indus. Appl.,, vol. 48, pp. 79-87, 2012. 